failure are progressive, it suggests that this counter-regulatory mechanism within the heart is limited and ultimately fails to provide long-term protection. Secondly, we must consider whether or not it will ultimately be beneficial or detrimental to treat (i.e., prevent) cardiac hypertrophy in response to pathologic conditions such as hypertension or valvular disease. In other words, would cardiac decompensation simply occur sooner if one blocks the hypertrophic phase of the response?

1. Lorell, B.H., and Carabello, B.A. 2000. Left ventricular hypertrophy: pathogenesis, detection, and prognosis. Circulation. 102:470-479.

2. Levy, D., Garrison, R.J., Savage, D.D., Kannel, W.B., and Castelli, W.P. 1990. Prognostic implications of echocardiographically determined left ventricular mass in the Framingham Heart Study. N. Engl. J. Med. 322:1561-1566.

3. Molkentin, J.D., and Dorn, G.W., II. 2001. Cytoplasmic signaling pathways that regulate cardiac hypertrophy. Annu. Rev. Physiol. 63:391-426.

4. Holtwick, R., et al. 2003. Pressure-independent cardiac hypertrophy in mice with cardiomyocyterestricted inactivation of the atrial natriuretic peptide receptor guanylyl cyclase-A.J. Clin. Invest. 111:1399-1407. doi:10.1172/JCI200317061.

5. Misono, K.S. 2002. Natriuretic peptide receptor: structure and signaling. Mol. Cell Biochem. 230:49-60.

6. Tremblay, J., Desjardins, R., Hum, D., Gutkowska, J., and Hamet, P. 2002. Biochemistry and physiology of the natriuretic peptide receptor guanylyl cyclases. Mol. Cell Biochem. 230:31-47.

7. Silberbach, M., and Roberts, C.T., Jr. 2001. Natriuretic peptide signaling: molecular and cellular pathways to growth regulation. Cell Signal. 13:221-231

8. Lainchbury, J.G., et al. 1997. Cardiac natriuretic peptides as predictors of mortality. J. Intern. Med. 241:257-259.

9. Klinger, J.R., et al. 1993. Cardiopulmonary responses to chronic hypoxia in transgenic mice that overexpress ANP. J. Appl. Physiol. 75:198-205.

10. Kishimoto, I., Rossi, K., and Garbers, D.L. 2001. A genetic model provides evidence that the receptor for atrial natriuretic peptide (guanylyl cyclase-A) inhibits cardiac ventricular myocyte hypertrophy. Proc. Natl. Acad. Sci. U. S. A 98:2703-2706.

11. Oliver, P.M., et al. 1997. Hypertension, cardiac hypertrophy, and sudden death in mice lacking natriuretic peptide receptor A. Proc. Natl. Acad. Sci. U. S. A. 94:14730-14735.

12. Lopez, M.J., et al. 1995. Salt-resistant hypertension in mice lacking the guanylyl cyclase-A receptor for atrial natriuretic peptide. Nature. 378:65-68.

13. Masciotra, S., Picard, S., and Deschepper, C.F. 1999. Cosegregation analysis in genetic crosses suggests a protective role for atrial natriuretic factor against ventricular hypertrophy. Circ. Res. 84:1453-1458.

14. Knowles, J.W., et al. 2001. Pressure-independent enhancement of cardiac hypertrophy in natriuretic peptide receptor A-deficient mice. J. Clin. Invest. 107:975-984.

15. Kuhn, M., et al. 2002. Progressive cardiac hypertrophy and dysfunction in atrial natriuretic peptide receptor (GC-A) deficient mice. Heart. 87:368-374

16. Ellmers, L.J., et al. 2002. Ventricular expression of natriuretic peptides in Npr1(-/-) mice with cardiac hypertrophy and fibrosis. Am. J. Physiol. Heart Circ. Physiol. 283:H707-H714.

17. Calderone, A., Thaik, C.M., Takahashi, N., Chang, D.L., and Colucci, W.S. 1998. Nitric oxide, atrial natriuretic peptide, and cyclic GMP inhibit the growth-promoting effects of norepinephrine in cardiac myocytes and fibroblasts. J. Clin. Invest. 101:812-818.

18. Silberbach, M., et al. 1999. Extracellular signalregulated protein kinase activation is required for the anti-hypertrophic effect of atrial natriuretic factor in neonatal rat ventricular myocytes. J. Biol. Chem. 274:24858-24864.

19. Horio, T., et al. 2000. Inhibitory regulation of hypertrophy by endogenous atrial natriuretic peptide in cultured cardiac myocytes. Hyperten sion. 35:19-24.

20. Fiedler, B., et al. 2002. Inhibition of calcineurinNFAT hypertrophy signaling by cGMP-dependent protein kinase type I in cardiac myocytes. Proc. Natl. Acad. Sci. U. S. A. 99:11363-11368

21. John, S.W., et al. 1995. Genetic decreases in atrial natriuretic peptide and salt-sensitive hypertension. Science. 267:679-681.

22. Keating, G.M., and Goa, K.L. 2003. Nesiritide: a review of its use in acute decompensated heart failure. Drugs. 63:47-70.

\title{
Double target for tumor mass destruction
}

\author{
Pipsa Saharinen and Kari Alitalo
}

Molecular/Cancer Biology Laboratory, Haartman Institute, Helsinki University Central Hospital and Biomedicum Helsinki, University of Helsinki, Helsinki, Finland

J. Clin. Invest. 111:1277-1280 (2003). doi:10.1172/JCI200318539.

As the growth of most cancers is dependent on the growth of tumor blood vessels, inhibition of tumor angiogenesis may provide an efficient strategy to slow down or block tumor growth. The possibility of selectively

\footnotetext{
Address correspondence to: Kari Alitalo, Molecular/Cancer Biology Laboratory, PO Box 63 (Haartmaninkatu 8), University of Helsinki, Helsinki, 00014 Finland.

Phone: 358-9-191 25511;

Fax: 358-9-191 25510;

E-mail: kari.alitalo@helsinki.fi.

Conflict of interest: The authors have declared that no conflict of interest exists.

Nonstandard abbreviations used: hypoxiainducible factor (HIF); endothelial cell (EC); pericyte (PC).
}

targeting angiogenic vasculature in a tumor mass depends on molecular, cellular, and structural differences between the tumor vessels and their normal counterparts (Figure 1). Tumor cells, like normal cells, need to be located close to the blood vessels serving their metabolic demands to the extent that in solid tumors every endothelial cell in a tumor vessel is considered to support several concentric layers of tumor cells (1). A hypoxic tumor generates its own microcirculation mainly via the hypoxia-inducible factor (HIF) complex, which is activated by inhibition of its oxygen-dependent prolyl hydroxylation and proteaso- mal destruction (2). This leads to increased transcription of several hypoxia-induced genes. One such gene encodes VEGF, which activates endothelial cell (EC) responses during angiogenesis in coordination with, for example, matrix adhesion events. Studies of the signaling pathways of VEGF receptors, integrins, and cadherins have provided new antiangiogenic strategies for inhibition of tumor growth, and inhibition of VEGF-C and VEGF-D signals that stimulate lymphangiogenesis seems to inhibit lymphatic metastasis in mice (3). One of the most striking new developments in antiangiogenesis research concerns the inflammatory cells and pericytes (PCs) associated with the tumor vasculature. The new findings presented in this issue of the JCI by Bergers and collaborators suggest that the $\mathrm{PCs}$ of tumor blood vessels and their signaling mechanisms via the PDGFR- $\beta$ are functionally important for the maintenance of tumor blood vessels (4). These findings add another constituent cell type of tumor stroma to the list of anticancer targets. 

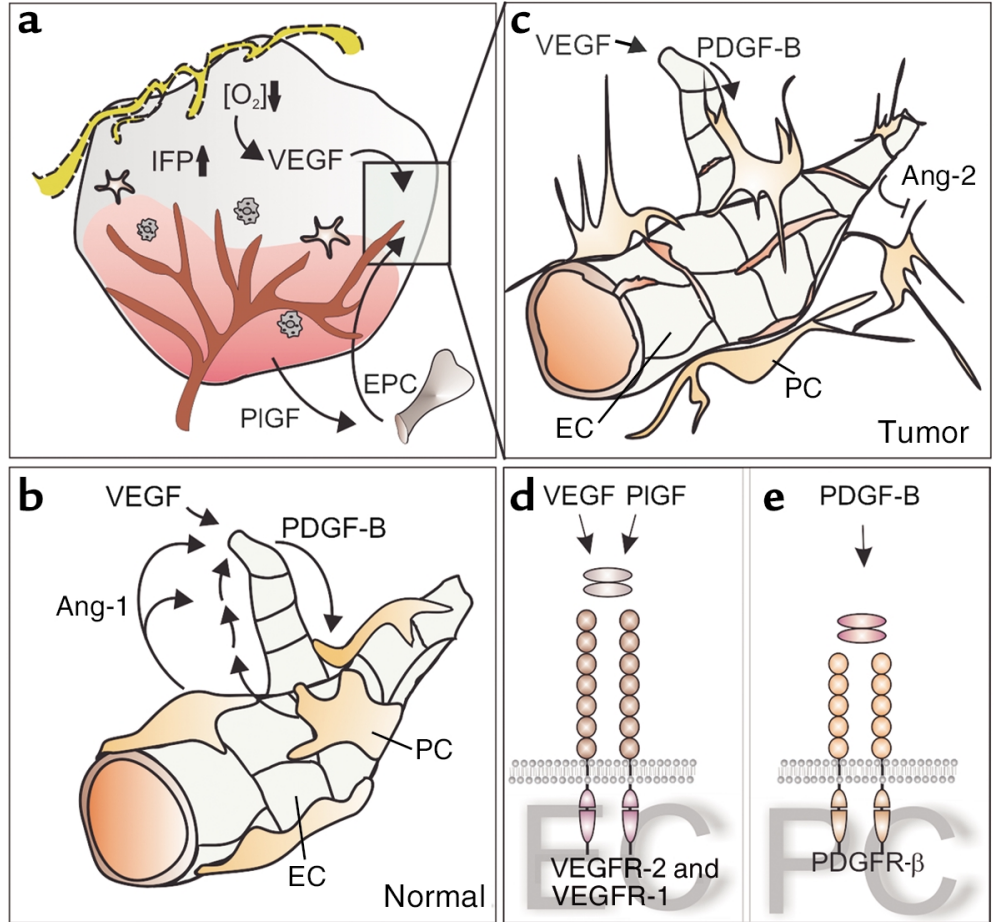

e

PDGF-B

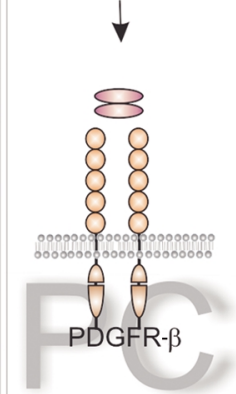

\section{Figure 1}

Growth factor receptor signals involved in tumor angiogenesis and vascular development. A solid tumor is dependent on new blood vessels for growth. (a) Angiogenesis is induced by VEGF produced mainly by hypoxic tumor cells and inflammatory cells (such as macrophages, which also secrete MMPs involved in the angiogenic switch) (19). Placental growth factor (PIGF) produced in the tumor may recruit bone marrow-derived endothelial precursor cells (EPCs), which become incorporated into the angiogenic vessels (20). The tip cells of a growing angiogenic sprout, and also the tumor cells, secrete PDGF-B, which binds to the EC surface and recruits PCs to migrate along the angiogenic sprouts $(8,9)$. In normal tissues, blood vascular ECs and PCs are in tight contact, and angiopoietin-1 (Ang-1) increases the stability of the vessels (b). In tumor vessels, Ang-2, an antagonist of Ang-1, is produced by the ECs, and the PCs are often only loosely attached to EC tubes; they also have long projections reaching out from the vessels to the surrounding matrix $(13,22)(\mathbf{c})$. The tumor blood vessels are leaky and the peritumoral lymphatic vessels (shown in yellow in panel a) cannot clear the extravasated material (10), which results in increased interstitial fluid pressure (IFP) within the tumor. The findings of Bergers et al. now suggest that concomitant inhibition of ECs and $\mathrm{PCs}$ through inhibition of their respective transmembrane tyrosine kinase receptors VEGFR-2 and VEGFR-1 (d) and PDGFR- $\beta$ (e) results in more efficient inhibition of angiogenesis (4). The roles of additional ligands, VEGF-B (23) and PDGF-D (24, 25), which bind to VEGFR- 1 and PDGFR- $\beta$, respectively, are not yet clear in these processes.

\section{Role of PDGF signals in vascular development}

The different types of blood vessels develop through the assembly of the two principal cell types - ECs and PCs (or vascular smooth muscle cells). ECs first form tubes, which subsequently recruit a $\mathrm{PC} /$ smooth muscle cell coating. PCs contribute to the stability of the capillary wall by participating in the assembly of the basal lamina beneath the endothelial layer, and they also appear to regulate EC function by, for example, promoting EC survival (5). A number of mouse mutants have shown that impairment of either the PC or EC population impacts the other cell population as well, indicating that the communication between ECs and PCs is essential for normal blood vessel development (6).

PDGF-B has been identified as an essential factor regulating PCs, and thus, indirectly, EC function. Targeted disruption of PDGF-B or PDGFR- $\beta$ in mice results in ablation of microvascular PCs, which normally form part of the capillary wall $(7,8)$. The mutant mouse embryos develop abnormal vasculature with an irregular vessel diam- eter, increased leakiness, and capillary microaneurysms. During normal development, PDGF-B secreted (and bound) by vascular ECs signals to neighboring PC progenitors expressing PDGFR- $\beta$ to promote the recruitment of PCs along the angiogenic sprouts. The sprouting capillaries in the PDGF-B knockout mice have been found unable to attract PDGFR$\beta$-positive PC progenitor cells, which leads to abnormal capillaries (7). Endothelium-restricted ablation of PDGF-B results in viable mice with extensive variation of density of PCs in the central nervous system and a range of retinal microvascular abnormalities reminiscent of those found in diabetic humans (9). Proliferative retinopathy developed when PC density was less than $50 \%$ of normal, indicating that a reduction of the PC density is sufficient to cause retinopathy in mice. This suggests that PC loss may also be a causal pathogenic event in human diabetic retinopathy.

\section{PDGFR as a second tumor target}

Confocal- and intravital microscopic imaging of tumor blood vessels have shown that the tumor vessels are structurally and functionally abnormal when compared with normal vessels. The tumor vessels are characterized by variable and often increased diameter, irregular shape, chaotic blood flow, and leakiness (10). The vascular ECs in tumors also have an altered pattern of gene expression when compared to ECs in normal tissue, indicating that the tumor ECs are distinct also at the molecular level (11). Such abnormalities of tumor vessels provide the potential for targeting these vessels without destroying the normal vasculature. Through probing of accessible EC surface molecules by in vivo phage display, a few endothelial molecules have been identified that characterize ECs from either tumor or normal tissue (12).

PCs show clear differences in their association with ECs between normal and tumor vessels, and considerable heterogeneity has been detected in the degree of PC coating of the angiogenic vessels in different types of tumors (13). In many tumors, PC coating of vessels is sparse compared to normal tissues. In two transplantable mouse tumor 
models, T241 fibrosarcoma and KRIB osteosarcoma, tumor vessels fail to recruit a proper coverage of PCs, although tumor vessel endothelium is found to express PDGF-B. The sparse PC coat of tumor vessels may result from a limited pool of PCs available for recruitment from nearby tissues or possibly also from the bone marrow (14). Even in tumors, where tumor vessels are coated with PCs, such as in the pancreatic tumors of the RIP1-Tag2 mice, $\mathrm{MCa}-\mathrm{IV}$, and Lewis lung tumors, the coating is abnormal; PCs are loosely associated with the ECs, with wide spaces separating the two cell types (13). PCs also show cytoplasmic processes penetrating into the tumor parenchyma and the basement membrane is only loosely associated with either ECs or PCs (13).

In this issue of the JCI, the transgenic RIP1-Tag2 pancreatic $\beta$-cell tumor model, where a clear angiogenic switch has been described to occur during tumorigenesis, has been used to investigate the role and potential use of PCs as a target of antiangiogenenic therapy (4). The study suggests that the inhibition of PDGFR- $\beta$ kinase activity by SU6668 or STI571/Gleevec is sufficient to inhibit tumor growth although the tumor cells themselves do not express PDGFR- $\beta$. Instead, PDGFR- $\beta$-positive cells aligning the tumor blood vessels may be the main common target of these tyrosine kinase inhibitors. The combination of SU6668 or STI571/Gleevec with the VEGFR inhibitor SU5416 was found to be most effective in reducing tumor growth, even in the late stages, where inhibition of VEGF signaling alone was inefficient. This result supports the previous suggestions that in the absence of PC coating the vascular ECs become sensitized to apoptosis after VEGF withdrawal (15). One caveat in the interpretation of these studies is that the PC recruitment and tumor growth reduced by SU6668 or STI571/Gleevec may not necessarily be causally linked as they may also relate to the inhibition of other kinases. Nevertheless, the study demonstrates the promise of combinatorial approaches to angiogenesis inhibition and should inspire further attempts to target the EC and PC compartments separately and in combination in experimental tumors.

\section{Implications for stromal targeting}

In tumors where oncogenic mutants of PDGFR- $\beta$ have been characterized, such as the TEL-PDGFR- $\beta$ fusion in chronic myelomonocytic leukemia or where ectopic induction of ligand secretion occurs, such as the secretion of the collagen-PDGF-B fusion in dermatofibrosarcoma protuberans, the targeting of PDGFR- $\beta$ is an obvious strategy for tumor therapy (16). The article by Bergers et al., however, has much wider implications because the targeting of PDGFR- $\beta$ in PCs can be beneficial even in the absence of oncogenic forms of the receptor (4). In a recent study, the blocking of PDGFR- $\beta$ by the STI571/Gleevec tyrosine kinase inhibitor or by nucleic acid aptamers was found to enhance tumor chemotherapeutic efficacy in mice (17). In the tumor type used in the study, the PDGFR- $\beta$ positive tumor stromal cells appeared to be the drug target. Inhibition of their PDGFR- $\beta$ was found to lower the otherwise high interstitial fluid pressure in the tumor thus enhancing drug uptake. The exact mechanisms of such an effect are not completely known, but they may be related to the finding that the PDGFR- $\beta$ signaling pathway is responsible for pericellular matrix contraction by connective tissue cells. PDGFR- $\beta$-induced activation of PI3K plays a role in control of interstitial fluid pressure in normal tissues as well (18).

It is becoming clear that in addition to the tumor cells themselves, the tumor microenvironment, including inflammatory cells and fibroblasts, has a critical role in the neoplastic process, promoting cell migration, proliferation, and survival (19). Many tumors secrete placental growth factor (20), as well as other cytokines and chemokines that are mitogenic and/or chemotactic for inflammatory cells, fibroblasts, and ECs. Tumor infiltrating macrophages, in turn, can secrete VEGF and VEGF-C/VEGF-D promoting angiogenesis and lymphangiogenesis, respectively $(3,21)$. While the tumor cells themselves may escape various therapeutic strategies by develop- ing resistant variants, the stromal cells they have co-opted may provide a new opportunity for therapeutic intervention through, for example, PDGFR- $\beta$ inhibitors or new anti-inflammatory therapeutic approaches. Indeed, it is possible that the phenotypic changes in tumor stromal cells are sufficiently stable and permanent for their use as targets of tumor growth inhibition. Isolation of distinct populations of stromal cells and their characterization by gene expression profiling would give important information for these targeting approaches. Furthermore, recognizing the complexity of tumorigenesis, and the interdependence between tumor cells and cells of the tumor microenvironment, targeting a single component may not be sufficient. Instead, as shown by the paper by Bergers et al., combinatorial strategies may provide improved efficacy for targeted destruction of the tumor mass (4).

1. Folkman, J. 2002. Role of angiogenesis in tumor growth and metastasis. Semin. Oncol. 29:15-18.

2. Pugh, C.W., and Ratcliffe, P.J. 2003. The von Hip pel-Lindau tumor suppressor, hypoxia-inducible factor-1 (HIF-1) degradation, and cancer pathogenesis. Semin. Cancer Biol. 13:83-89.

3. Alitalo, K., and Carmeliet, P. 2002. Molecular mechanisms of lymphangiogenesis in health and disease. Cancer Cell. 1:219-227.

4. Bergers, G., Song, S., Meyer-Morse, N., Bergsland, E., and Hanahan, D. 2003. Benefits of targeting both pericytes and endothelial cells in the tumor vasculature with kinase inhibitors. J. Clin. Invest. 111:1287-1295. doi:10.1172/JCI200317929.

5. Benjamin, L.E., Hemo, I., and Keshet, E. 1998. A plasticity window for blood vessel remodeling is defined by pericyte coverage of the preformed endothelial network and is regulated by PDGF-B and VEGF. Development. 125:1591-1598.

6. Beck, L., Jr, and D'Amore, P.A. 1997. Vascular development: cellular and molecular regulation. FASEB J. 11:365-373

7. Lindahl, P., Johansson, B.R., Leveen, P., and Betsholtz, C. 1997. Pericyte loss and microaneurysm formation in PDGF-B-deficient mice. Science. 277:242-245.

8. Hellstrom, M., Kalen, M., Lindahl, P., Abramsson, A., and Betsholtz, C. 1999. Role of PDGF-B and PDGFR-beta in recruitment of vascular smooth muscle cells and pericytes during embryonic blood vessel formation in the mouse. Devel opment. 126:3047-3055.

9. Enge, M., et al. 2002. Endothelium-specific platelet-derived growth factor-B ablation mimics diabetic retinopathy. EMBO J. 21:4307-4316.

10. Jain, R.K., Munn, L.L., and Fukumura, D. 2002 Dissecting tumour pathophysiology using intravital microscopy. Nat. Rev. Cancer. 2:266-276.

11. St. Croix, B., et al. 2000. Genes expressed in human tumor endothelium. Science. 289:1197-1202.

12. Ruoslahti, E. 2002. Specialization of tumour vasculature. Nat. Rev. Cancer. 2:83-90.

13. Morikawa, S., et al. 2002. Abnormalities in pericytes on blood vessels and endothelial sprouts in tumors. Am. J. Pathol. 160:985-1000. 
14. Abramsson, A., et al. 2002. Analysis of mural cell recruitment to tumor vessels. Circulation. 105:112-117.

15. Benjamin, L.E., Golijanin, D., Itin, A., Pode, D. and Keshet, E. 1999. Selective ablation of immature blood vessels in established human tumors follows vascular endothelial growth factor withdrawal. J. Clin. Invest. 103:159-165.

16. Simon, M.P., et al. 1997. Deregulation of the platelet-derived growth factor B-chain gene via fusion with collagen gene COL1A1 in dermatofibrosarcoma protuberans and giant-cell fibroblastoma. Nat. Genet. 15:95-98.

17. Pietras, K., et al. 2002. Inhibition of PDGF receptor signaling in tumor stroma enhances antitumor effect of chemotherapy. Cancer Res. 62:5476-5484

18. Heuchel, R., et al. 1999. Platelet-derived growth factor beta receptor regulates interstitial fluid homeostasis through phosphatidylinositol-3' kinase signaling. Proc. Natl. Acad. Sci. U. S. A. 96:11410-11415.

19. Coussens, L.M., and Werb, Z. 2002. Inflammation and cancer. Nature. 420:860-867.

20. Luttun, A., et al. 2002. Revascularization of ischemic tissues by PIGF treatment, and inhibition of tumor angiogenesis, arthritis and atherosclerosis by anti-Flt1. Nat. Med. 8:831-840

21. Schoppmann, S.F., et al. 2002. Tumor-associated macrophages express lymphatic endothelial growth factors and are related to peritumoral lymphangiogenesis. Am. J. Pathol. 161:947-956 22. Yancolpoulos, G.D., et al. 2000. Vascular-specific growth factors and blood vessel formation. Nature. 407:242-248.

23. Olofsson, B., et al. 1998. Vascular endothelial growth factor B (VEGF-B) binds to VEGF receptor- 1 and regulates plasminogen activator activity in endothelial cells. Proc. Natl. Acad. Sci.U. S. A. 95:11709-11714.

24. Bergsten, E., et al. 2001. PDGF-D is a specific, protease-activated ligand for the PDGF- $\beta$-receptor. Nat. Cell Biol. 3:512-516.

25. LaRochelle, W.J., et al. 2001. PDGF-D, a new protease-activated growth factor. Nat. Cell Biol. 3:517-521

\title{
Peptide-based treatment for autoimmune diseases: learning how to handle a double-edged sword
}

\author{
Alberto Pugliese \\ Departments of Medicine, and Immunology and Microbiology, Immunogenetics Program, \\ Diabetes Research Institute, University of Miami, Miami, Florida, USA
}

J. Clin. Invest. 111:1280-1282 (2003). doi:10.1172/JCI200318395.

Several self-molecules have been identified as target antigens in autoimmune diseases. Since lack or loss of tolerance to these molecules is one of the key events promoting autoimmunity, researchers are exploring the possibility that the administration of antigens or peptides may stimulate tolerogenic mechanisms and delay or prevent the full phenotypic expression of autoimmune diseases. There is much enthusiasm for such therapies, as these will probably be disease-specific and not associated with the side effects of conventional immunosuppression. Studies have been performed and are ongoing in both rodents and humans,

\footnotetext{
Address correspondence to: Alberto Pugliese, Departments of Medicine, and Immunology and Microbiology, Immunogenetics Program, Diabetes Research Institute, University of Miami, $1450 \mathrm{NW}$

Tenth Avenue, Miami, Florida 33136, USA. Phone (305) 243-5348; Fax: (305) 243-4404;

E-mail: apuglies@med.miami.edu.

Conflict of interest: The author has declared that no conflict of interest exists.

Nonstandard abbreviations used: type 1 diabetes (T1D); glutamic acid decarboxylase (GAD); Diabetes Prevention

Trial-Type 1 (DPT-1).
}

using whole antigens or peptides, and testing diverse administration routes such as intrathymic, intraperitoneal, intravenous, subcutaneous, oral, and intranasal. Despite many studies, robust data demonstrating clinical benefits are not yet available (1).

\section{Antigen and/or peptide-based interventions in diabetes}

Type 1 diabetes (T1D) represents one of the most suitable diseases to exemplify such heterogeneous outcomes. Three autoantigens - proinsulin/ insulin, glutamic acid decarboxylase (GAD), and tyrosine phosphatase-like protein IA-2 (or ICA512) - have been well characterized in both humans and the NOD mouse model of autoimmune diabetes (2). Although all of these molecules are expressed in pancreatic islets, insulin and its precursor proinsulin are uniquely secreted by pancreatic $\beta$-cells. Several studies have suggested an important role for autoimmune responses to epitopes of insulin/proinsulin, such as the peptides B9-23 (the 9-23 amino acid region of the insulin $B$ chain) $(3,4)$ and B24-C36 (the proinsulin B-chain-
C-peptide junction) $(5,6)$. In NOD mice, both subcutaneous and oral administration of insulin can delay or prevent diabetes; oral insulin induces regulatory $\mathrm{CD}^{+}{ }^{+} \mathrm{T}$ cells, while nasorespiratory insulin induces regulatory $\mathrm{CD}^{+} \gamma \delta$-T cells $(7-11)$. However, in a Diabetes Prevention Trial-Type 1 (DPT-1) study that involved the parenteral administration of insulin, no significant effect on progression to overt disease in autoantibody-positive first-degree relatives of T1D patients, who have increased risk of developing diabetes, was demonstrated (12). While the results of the oral insulin arm of DPT-1 are expected in June 2003, a randomized, crossover, pilot trial of intranasal insulin in at-risk first-degree relatives demonstrated changes in immune and metabolic markers that were consistent with an immunoprotective effect (13).

The subcutaneous or intranasal administration of the insulin peptide B9-23 can also prevent diabetes in NOD mice (3), similarly to the neonatal administration of the B10-24 peptide (9). However, the administration of several antigen-derived peptides, in adjuvant, to newborn NOD mice, resulted in the early activation of multiple autoimmune responses (14). Similarly, the intrathymic injection of T1D-associated antigens or peptides resulted in delayed or accelerated diabetogenesis, depending on the peptides used (15). Recent studies in mice have also shown that the repeated administration of insulin or GAD peptides, including the B9-23 peptide, can induce lethal anaphylactic responses $(16,17)$. While similar occurrences have not been reported in a phase I trial in which a modified insulin B9-23 peptide (altered peptide 